\title{
Long-term results of protocol kidney biopsy directing steroid withdrawal in simultaneous pancreas-kidney transplant patients
}

\author{
Nemin Zhu ${ }^{1}$; Neal E. Rowe ${ }^{2}$; Paul R. Martin ${ }^{1,3}$; Sean S. Luke ${ }^{1}$; Thomas B. McGregor ${ }^{4}$; Frank \\ Myslik $^{1}$; Vivian C. McAlister ${ }^{1,3,5}$; Alp Sener ${ }^{1,3,5}$; Patrick P. Luke ${ }^{1,3,5}$ \\ ${ }^{1}$ Western University Schulich School of Medicine and Dentistry, London, ON; ${ }^{2}$ Department of Surgery, University of \\ Ottawa, Ottawa, ON; ${ }^{3}$ Department of Surgery, London Health Sciences Centre, Western University, London, \\ ON; ${ }^{4}$ Department of Surgery, Queen’s University, Kingston, ON; ${ }^{5}$ Multi-Organ Transplant Program, London Health \\ Sciences Centre, London, ON; Canada
}

Cite as: Can Urol Assoc J 2018 Feb. 23; Epub ahead of print. http://dx.doi.org/10.5489/cuaj.4702

\section{Published online February 23, 2018}

$* * *$

\section{Abstract}

Introduction: We sought to determine whether protocol biopsies could be used to guide treatment and improve outcomes in simultaneous pancreas-kidney (SPK) patients.

Methods: Between 2004 and 2013, protocol biopsies were performed on SPK patients at 3-6 months and one year post-transplant. Maintenance immunosuppression consisted of a calcineurin inhibitor, anti-proliferative agent, and corticosteroid. Corticosteroid was withdrawn in negative early biopsies, maintained in subclinical/borderline biopsies, and increased if Banff IB or greater rejection was identified. Endpoints included presence of interstitial fibrosis and tubular atrophy on biopsy at one year (IF/TA), rejection episodes, renal and pancreas function at five years’ followup.

Results: Forty-one SPK transplant patients were reviewed and a total of 75 protocol biopsies were identified. On early biopsy, 51\% had negative biopsies, 44\% had borderline rejection, and 5\% had subclinical rejection. Renal and pancreas function were not significantly different at one, two, and five years post-transplant between negative vs. borderline early biopsy patients. No difference in the degree of IF/TA was found between these two groups.

Conclusions: To our knowledge, this is the first study to evaluate protocol biopsies as an investigative tool prior to steroid withdrawal in SPK patients. Our study suggests that there are no detrimental functional or histological effects at five years post-transplant, despite weaning steroids in the negative biopsy group. 


\section{Introduction}

Interstitial fibrosis and tubular atrophy (IF/TA) is the principle cause of kidney allograft failure and is commonly precipitated by episodes of acute rejection. ${ }^{1}$ While recent improvements in immunosuppressive therapy have reduced the incidence of acute rejection, these pharmacologic advances have not translated into a reduction in the prevalence of IF/TA or an improvement in longterm outcomes. ${ }^{2,3}$ Current literature suggests that clinically undetectable graft pathology (including borderline and subclinical rejection) may be a predictor of future development of IF/TA and eventual graft loss. $^{3-5}$

Subclinical rejection refers to a histologic finding of acute cellular rejection using the Banff criteria ( $\geq$ grade IA) in the absence of renal dysfunction. ${ }^{3,6}$ Borderline rejection is defined as Banff grade < IA in the absence of renal dysfunction. While some authors consider borderline rejection to be grouped with subclinical rejection, the significance of borderline rejection is largely unknown. ${ }^{3}$ Protocol biopsies are histologic allograft samples taken at predetermined times after transplant, in the absence of any clinically detectable graft dysfunction. These biopsies provide the opportunity to recognize silent graft pathology (subclinical/borderline changes) in a timely manner and potentially optimize treatment to prolong graft function and survival. A study by Nankivell et al. demonstrated a high incidence of IF/TA in simultaneous pancreas-kidney (SPK) patients undergoing protocol

biopsies. ${ }^{1}$ However, these biopsies were not used to guide subsequent treatment of patients. Steroid withdrawal has been controversial in SPK patients, as more antiquated studies suggest that rejection rates and renal function are potentially impacted if steroids are withdrawn in all patients irrespective of rejection risk. ${ }^{7}$ In our cohort of SPK patients, we aimed to wean corticosteroids in patients with an absence of subclinical or borderline rejection at 3 months, in order to reduce steroidrelated complications. The overall aim of this study was to determine whether identification of subclinical pathology with protocol biopsies could be used to select SPK patients for safe steroid withdrawal.

\section{Methods}

\section{Patients}

Institutional review board approved our study. The study group consisted of 61 consecutive SPK transplant recipients. All recipient surgeries occurred at the London Health Sciences Center (LHSC) between January 2004 and August 2013. Biopsies were read at LHSC by the Pathology Department. Patients were followed for a minimum of 5 years post-transplant. Protocol biopsies were performed on all SPK patients at 3-6 months. Exclusion criteria included 1) patient declining protocol biopsy (4 patients), 2) patient non-compliance (4 patients), 3) early graft loss (7 patients), and 4) post-operative anticoagulation therapy (5 patients).

\section{Immunotherapy}

All SPK patients received anti-thymocyte globulin (total 5-8 mg/kg) for induction immunosuppression and continued on 3-drug maintenance immunosuppression (combination of calcineurin inhibitor (tacrolimus), anti-proliferative agent (mycophenolate mofetil or sirolimus), and corticosteroid). Target tacrolimus levels were $5-8 \mathrm{ng} / \mathrm{ml}$ in the first 3 months and 3-5 ng/ml 
thereafter. Methylprednisolone was initiated at $250 \mathrm{mg}$ IV, reduced to $1 \mathrm{mg} / \mathrm{kg} \mathrm{IV}$, and reduced daily by $10 \mathrm{mg}$ until oral prednisone was administered at $5 \mathrm{mg}$ daily.

\section{Protocol biopsies}

The early 3-6 month protocol biopsy determined the decision to alter corticosteroid therapy. Biopsies were performed under ultrasound guidance with a Monopty biopsy gun (Bard ${ }^{\circ}$, Covington GA). The biopsy was adequate if a minimum of 2 full cores with a total of $>8$ glomeruli were available for assessment under microscopy. If there was no sign of rejection on the biopsy, the prednisone dose was tapered and withdrawn over 2 weeks. If there was borderline rejection on the biopsy, then prednisone therapy was maintained at $5 \mathrm{mg}$ daily. If Banff IB or greater rejection was identified, patients were treated with methylprednisolone boluses $(250 \mathrm{mg}$ then $1 \mathrm{mg} / \mathrm{kg}$ the next day with reduction of $10 \mathrm{mg}$ daily until a dose of $5 \mathrm{mg}$ daily was achieved). Repeat biopsy within 9 months was performed to determine presence or absence of rejection or IF/TA.

Protocol biopsies were not performed on the pancreas graft due to technical difficulties in visualizing the pancreas and risk of pancreatitis or bleeding. As well, assessment of post-transplant donor specific antibodies was not performed in our patients and is currently not part of our posttransplant monitoring protocol.

\section{Outcomes}

The outcomes measured included creatinine, eGFR, presence of IF/TA, number of rejection episodes, and HbA1c.

\section{Statistics}

Statistical analysis was carried out using GraphPad Prism 6.0 software (GraphPad Software, Inc. 7825 Fay Avenue, Suite 230 La Jolla, CA 92037 USA). T- test was used for the comparison of means and standard deviations of the two groups. Log-rank (Mantel-Cox) test was used to compare graft and patient survival. Analysis was intention to treat based on their initial early protocol biopsy. Differences were considered significant when $\mathrm{p}<0.05$.

\section{Results}

\section{Patients and graft survival}

Forty one consecutive SPK transplant patients were reviewed. Donor and recipient characteristics were similar in between the steroid withdrawal group (no rejection on protocol biopsy) and the group maintained on steroids (borderline or acute rejection). Demographics are displayed in Tables 1 and 2. The 5-year death-censored renal graft survival rate was 95\% in the steroid withdrawal and 100\% in the maintenance group ( $\mathrm{p}=\mathrm{NS}$ ). The graft losses were secondary to infections and non-compliance. The 5-year death-censored pancreas graft survival rates were identical at 85\% in each group. Graft losses were secondary to non-compliance, infections as well as development of post-transplant diabetes mellitus from obesity.

\section{Rejection}

A total of 41 early protocol biopsies were performed in 41 patients between 3 and 6 months. Twenty six out of 41 patients had 1-year follow-up protocol biopsies that were adequate for analysis; 12 out 
of 41 patients had 16 additional protocol biopsies beyond the follow-up biopsy. Overall, a total of 85 protocol biopsies were performed. In addition to protocol biopsies, 24 out of 41 patients underwent for-cause biopsies for allograft dysfunction at some point during the 5 years of follow-up.

Fifty one percent of patients had no histological changes on early protocol biopsy (negative biopsy group) and prednisone therapy was withdrawn. The 1-year protocol biopsy of these patients ( $\mathrm{n}=14)$ showed that 57\% remained negative, 36\% developed borderline rejection and 7\% developed Banff IB rejection. Patients who later developed borderline rejection were restarted on steroid therapy. Forty-four percent of patients had borderline changes on early protocol biopsy (borderline biopsy group) and prednisone therapy was maintained. The 1-year protocol biopsy of these patients $(n=11)$ showed that 36\% were negative, 55\% remained borderline and 9\% developed C4d+/DSA+ subclinical humoral changes. Refer to Figure 1 for visual diagram.

Of note, IFTA was quantified using the Banff classification. Grade I represented mild IFTA and grade II represented moderate IFTA. There was only one case of grade II IFTA on protocol biopsy. The remaining protocol biopsies were grade I IFTA.

There was no difference in the percentage of IF/TA between the negative vs. borderline biopsy group at 1 -year ( $56 \%$ vs. $50 \%, p=0.81$ ) or at any protocol biopsy time point ( $67 \%$ vs. $67 \%$, $p=1)$. There was also no difference in the number of clinical rejection episodes in the 5 years of follow-up between the two groups ( $17 \%$ vs. $25 \%, \mathrm{p}=0.62)$.

Two early protocol biopsies (5\%) demonstrated subclinical rejection (Banff IA). The first patient received prednisone maintenance successfully but refused any further protocol biopsy and had excellent renal and pancreatic graft function at 5 years. The second patient had a biopsy demonstrating Banff IA rejection. He was successfully treated with prednisone bolus therapy. He later developed Banff IIA rejection with C4d+/DSA+ antibody-mediated rejection at 18 months, demonstrated on follow-up protocol biopsy, despite having excellent graft function (creatinine 105 $\mu \mathrm{mol} / \mathrm{L}, \mathrm{HbA1c}$ 0.056). He was treated with plasmapheresis/intravenous immunoglobulin $2 \mathrm{~g} / \mathrm{kg}$ and prednisone therapy but lost his renal allograft from ongoing rejection 4 years post-transplant. He remains insulin-independent. One patient in the borderline group had a rising amylase despite having a stable serum creatinine at 9 months post-operatively. This patient had a negative renal biopsy, but since the amylase remained elevated, the pancreas was biopsied one week later; this showed no evidence of rejection.

Four patients were excluded from the study because they declined the early protocol biopsy. Three of these patients received a biopsy at 1 year which showed borderline changes. One of these patients subsequently developed AMR at 6 months. The fourth patient never received any protocol biopsies. He later developed IFTA on a for cause biopsy at 11 months.

\section{Graft function and survival}

The 3-month (89 $\mu \mathrm{mol} / \mathrm{L}$ vs. $105 \mu \mathrm{mol} / \mathrm{L}, \mathrm{p}=0.05)$ and 2-year $(90 \mu \mathrm{mol} / \mathrm{L}$ vs. $105 \mu \mathrm{mol} / \mathrm{L}, \mathrm{p}=0.04)$ post-transplant serum creatinine was statistically lower in the negative vs. borderline biopsy group (Table 3). However, the 5-year post-transplant serum creatinine was not significantly different between the two groups. More importantly, there was no difference in the eGFR as calculated by the Chronic Kidney Disease Epidemiology Collaboration (CKD-EPI) equation between the two groups at 3 month (90 vs. $\left.80 \mathrm{ml} / \mathrm{min} / 1.72 \mathrm{~m}^{2}, \mathrm{p}=0.21\right), 1$ (75 vs. $\left.76 \mathrm{ml} / \mathrm{min} / 1.72 \mathrm{~m}^{2}, \mathrm{p}=0.85\right), 2$ (82 vs. 75 
$\mathrm{ml} / \mathrm{min} / 1.72 \mathrm{~m}^{2}, \mathrm{p}=0.53$ ), and 5 years (65 vs. $55 \mathrm{ml} / \mathrm{min} / 1.72 \mathrm{~m}^{2}, \mathrm{p}=0.91$ ) post-transplant. The 5-year kidney graft survival was $95 \%$ and $100 \%$ in the negative and borderline biopsy groups respectively $(\mathrm{p}=\mathrm{NS})$.

In terms of pancreatic transplant function, both groups displayed similar HbA1c levels at 3 month ( 0.053 vs. $0.056, p=$ NS), 1 (0.053 vs. $0.058, p=0.05)$ and 5 years $(0.053$ vs. $0.048, p=N S)$ post-transplant. The \% of patients who remained insulin-free were the same in both groups at 5 years (Table 4).

\section{Steroid related complications}

We did not detect a difference in common steroid-related complications, including impaired glucose tolerance, hypertension, and dyslipidemia between the negative and borderline biopsy groups (Table 5). As well, none of the patients developed avascular necrosis of the hips and 4 patients in each group had cataract surgeries in the 5 years following transplantation.

\section{Discussion}

In this study, $44 \%$ of patients had early protocol biopsies demonstrating borderline rejection and 5\% had subclinical rejection. These results are consistent with the findings of Nankivell et al., who demonstrated subclinical rejection (acute and borderline) in $45.7 \%$ of patients at 3 months. ${ }^{1}$ In addition, our protocol biopsies demonstrated a high percentage of IF/TA at 1 year. The ability to detect subclinical rejection and IF/TA in kidney-only transplant patients had previously been shown to allow early treatment that may theoretically prolong graft function and survival. ${ }^{6,8}$ Choi et al. demonstrated that subclinical rejection detected at 2 weeks post-transplant was associated with a $30 \%$ decrease in graft survival at 10 years compared to those with normal findings. ${ }^{8}$ A small randomized control trial by Rush et al. showed that treatment of subclinical rejection detected by protocol biopsies lead to better histologic and functional outcomes in renal transplant patients compared to patients with no protocol biopsies. ${ }^{6}$ Recent findings from Loupy et al. showed that there are two distinct phenotypes of subclinical rejection, T cell-mediated rejection (TCMR) and antibodymediated rejection (ABMR) which are associated with distinct outcomes. ${ }^{9}$ Specifically, subclinical ABMR detected on 1-year protocol biopsy was associated with the poorest graft survival compared to the subclinical TCMR or negative biopsy groups. ${ }^{9}$ The distinction of subclinical TCMR and ABMR was not within the scope of our study.

The use of corticosteroids is an integral part of immunosuppressive therapy but is also associated with multiple adverse effects, including bone demineralization, weight gain, impaired glucose tolerance, and heart disease. ${ }^{10}$ Therefore, steroid withdrawal is a worthwhile goal in the management of SPK patients post-transplant. However, historic publications have indicated that steroid withdrawal may increase rejection and inferior long-term survival in renal transplant recipients. ${ }^{7}$ In our study, the early protocol biopsy was used to determine whether steroid therapy could be withdrawn in those patients that are at low risk for subsequent acute/chronic rejection. We assumed that patients with a negative early protocol biopsy represented a 'low risk' population. In these patients, steroid withdrawal did not lead to increased histopathologic changes at 1 year compared to borderline/subclinical biopsy patients who were maintained on steroids. There was also no difference in the eGFR or HbA1c between the two groups at 5 years post-transplant. This suggests that there are no functional detrimental effects despite weaning steroids in the negative early 
biopsy group. Therefore, the early protocol biopsy identified a group in whom steroids therapy could be successfully weaned. Compared with early steroid withdrawal (within 1 week) in all patients, minimal steroid exposure is not achieved using our approach, which may have limited our ability to reduce all steroid-related compliations. Our biopsy approach, therefore, offers a compromise between early withdrawal and steroid maintenance strategies.

Unlike subclinical rejection in which treatment is generally supported, the clinical benefit of treating borderline rejection is less clear. Roberts et al. reported that early biopsy showing borderline changes go on to develop clinical rejection if left untreated. ${ }^{11}$ Nankivell et al. showed that patients with borderline rejection had subsequent biopsies showing higher grades of chronic rejection. ${ }^{1}$ Herein, we did not detect a difference between the negative vs. borderline biopsy groups in the number of patients with IF/TA at 1 year and the number of clinical rejection episodes at 5 years posttransplant. This finding suggests that low dose prednisone may be beneficial in preventing worsening of IF/TA in the borderline rejection group. Greater numbers are needed to determine whether modification of immunotherapy (steroid boluses, increasing immunotherapy dose) of this 'at risk' group will reduce IF/TA and improve long term graft survival.

In our protocol biopsies, only the kidney transplant is biopsied. Our steroid withdrawal protocol assumes that concordant rejections occur within both kidney and pancreatic allografts. However, it is believed that there may be a significant discordance between renal biopsies and pancreatic allograft biopsies with regards to the detection of rejection.$^{12}$ As the clinical impact of protocol biopsies of the pancreas transplant has not been studied, it is unknown whether discordance in subclinical rejections can also occur in pancreas and kidney allografts.

There are a few limitations to this study. First, this was a retrospective chart review and patients were not randomized to steroid withdrawal vs. maintaining steroid therapy in either group. Therefore, the true significance of steroid withdrawal on renal function and histology of biopsy negative patients is unknown, and potentially, the rates of IF/TA could have been reduced if corticosteroids were not withdrawn on the biopsy negative group. Importantly, the number of patients included in this study was small, and the impact of steroid withdrawal on steroid-related factors was beyond the scope of the study. These weaknesses were balanced by the long follow-up of a minimum of 5 years in our cohorts. We recommend a study utilizing greater patient numbers in order to determine the optimal approach in treating borderline rejection discovered on protocol biopsy in SPK patients.

\section{Conclusion}

To our knowledge, this is the first study to evaluate the role of protocol biopsies in guiding steroid withdrawal for SPK transplant patients. Our study suggests weaning steroids in the negative early biopsy group does not lead to worse functional or histological pathology at 5 years post-transplant vs. the borderline group. Further investigations are needed to determine the significance of borderline rejection and whether immunotherapeutic modifications have any impact upon IF/TA. 


\section{References}

1. Nankivell B, Borrows R, Fung C, et al. The natural history of chronic allograft nephropathy. N Engl J Med 2003;349:2326-33.

2. Rush D. Protocol transplant biopsies: an underutilized tool in kidney transplantation. Clin J Am Soc Nephro 2006;1:138-43.

3. Gloor J, Cohen A, Lager D, et al. Subclinical rejection in tacrolimus treated renal transplant recipients. Transplantation 2002;73:1965-7.

4. Rush D. Protocol biopsies should be part of the routine management of kidney transplant recipients. Am J Kidney Dis 2002;40:671-3.

5. Kee T, Chapman J, O'Connell P, et al. Treatment of subclinical rejection diagnosed by protocol biopsy of kidney transplants. Transplantation 2006;82:36-42.

6. Rush D, Nickerson P, Gough J, et al. Beneficial effects of treatment of early subclinical rejection: a randomized study. J Am Soc Nephrol 1998;9:2129-34.

7. Sinclair N. Low-dose steroid therapy in cyclosporine-treated renal transplant recipients with well-functioning grafts. Canadian Medical Association Journal 1992; 147: p. 645-7.

8. Choi B, Shin M, Shin S, et al. Clinical significance of an early protocol biopsy in livingdonor renal transplantation: ten-year experience at a single center. Am J Transplant 2005;5:1354-60. 9. Loupy A, Vernerey D, Tinel C, et al. Subclinical rejection phenotypes at 1 year posttransplant and outcome of kidney allografts. J Am Soc Nephrol 2015;26:1721-31.

10. Moghadam-Kia S, Werth VP. Prevention and treatment of systemic glucocorticoid side effects. Int J Dermatol 2010;49:239-48.

11. Roberts I, Reddy S, Russell C, et al. Subclinical rejection and borderline changes in early protocol biopsy specimens after renal transplantation. Transplantation 2004;77:1194-8.

12. Troxell ML, Koslin DB, Norman D. Pancreas allograft rejection: analysis of concurrent renal allograft biopsies and post-therapy follow-up biopsies. Transplantation 2010; 90: 75-84. 


\section{Figures and Tables}

\begin{tabular}{|l|c|c|c|}
\hline Table 1. Donor demographics & \multicolumn{2}{l|}{} \\
\hline Characteristic & $\begin{array}{c}\text { Negative biopsy (steroid } \\
\text { withdrawal) }\end{array}$ & $\begin{array}{c}\text { Positive biopsy (steroid } \\
\text { maintained) }\end{array}$ & p \\
\hline Age & $31.8 \pm 12.3$ & $29.4 \pm 10.7$ & NS \\
\hline Kidney CIT & $5.78 \pm 2.47$ & $6.96 \pm 2.58$ & NS \\
\hline Pancreas CIT & $6.47 \pm 2.60$ & $8.13 \pm 3.06$ & NS \\
\hline BMI $\left(\mathrm{kg} / \mathrm{m}^{2}\right)$ & $25.85 \pm 2.99$ & $25.6 \pm 4.2$ & NS \\
\hline
\end{tabular}

Data are mean \pm standard deviation. BMI: body mass index; CIT: cold ischemic time.

\begin{tabular}{|l|c|c|c|}
\hline \multicolumn{1}{|c|}{ Table 2. Recipient demographics } & \\
\hline Characteristic & $\begin{array}{c}\text { Negative biopsy (steroid } \\
\text { withdrawal) }\end{array}$ & $\begin{array}{c}\text { Positive biopsy (steroid } \\
\text { maintained) }\end{array}$ & p \\
\hline Age & $43.8 \pm 8.70$ & $41.9 \pm 9.13$ & NS \\
\hline Sex (male \%) & $55 \%$ & $65 \%$ & NS \\
\hline $\begin{array}{l}\text { Race (Caucasian } \\
\%)\end{array}$ & $95 \%$ & $100 \%$ & \\
\hline $\begin{array}{l}\text { Transplant } \\
\text { number } \\
\text { (first \%) }\end{array}$ & $100 \%$ & & $100 \%$ \\
\hline PRA \% & $0.7 \pm 2.8$ & $1.2 \pm 3.4$ & NS \\
\hline Dialysis (\% HD) & $60 \%$ & $71 \%$ & NS \\
\hline
\end{tabular}

Data are mean \pm standard deviation. HD: preoperative mode of dialysis = hemodialysis; PRA\%: \% panel reactive antibody. 
Table 3. Renal function and survival for negative and borderline early protocol biopsies

\begin{tabular}{|l|c|c|c|c|}
\hline & & $\begin{array}{c}\text { Negative (steroid } \\
\text { withdrawal) }\end{array}$ & $\begin{array}{c}\text { Positive (steroid } \\
\text { maintained) }\end{array}$ & p \\
\hline Mean Cr & 3 months & $89 \pm 16$ & $105 \pm 30$ & 0.05 \\
\hline & 1 year & $97 \pm 17$ & $107 \pm 20$ & 0.04 \\
\hline & 2 years & $90 \pm 14$ & $105 \pm 29$ & 0.04 \\
\hline & 5 years & $92 \pm 14$ & $113 \pm 15$ & 0.01 \\
\hline & & & & NS \\
\hline & 3 months & $90 \pm 21$ & $80 \pm 20$ & NS \\
\hline & 1 year & $75 \pm 16$ & $76 \pm 15$ & NS \\
\hline & 2 years & $82 \pm 22$ & $75 \pm 18$ & NS \\
\hline & 5 years & $65 \pm 9$ & $55 \pm 18$ & NS \\
\hline
\end{tabular}

Data are means \pm standard deviation. CR: creatinine; eGRR: estimated glomerular filtration rate; NS: non-significant.

\begin{tabular}{|c|c|c|c|c|}
\hline & & $\begin{array}{c}\text { Negative } \\
\text { (steroid } \\
\text { withdrawal) }\end{array}$ & $\begin{array}{c}\text { Borderline } \\
\text { (steroid } \\
\text { maintained) }\end{array}$ & $\mathbf{p}$ \\
\hline \multirow[t]{3}{*}{ Mean HbA1c } & 3 months & $0.053 \pm 0.009$ & $0.056 \pm 0.008$ & NS \\
\hline & 1 year & $0.052 \pm 0.005$ & $0.058 \pm 0.009$ & 0.05 \\
\hline & 5 years & $0.053 \pm 0.006$ & $0.048 \pm 0.004$ & NS \\
\hline $\begin{array}{l}\text { 5-year graft } \\
\text { survival }\end{array}$ & & $85 \%$ & $85 \%$ & NS \\
\hline
\end{tabular}

Data are means \pm standard deviation. Pancreas graft survival is indicated by recipients being insulinfree. HbA1c: hemoglobin A1c; NS: non-significant. 


\begin{tabular}{|c|c|c|c|}
\hline & $\begin{array}{c}\text { LDL pre-transplant } \\
(\mathrm{mmol} / \mathrm{L})\end{array}$ & $\begin{array}{l}\text { LDL } 1 \text { year post- } \\
\text { transplant }\end{array}$ & $\mathbf{p}$ \\
\hline $\begin{array}{l}\text { Negative (steroid } \\
\text { withdrawal) }\end{array}$ & $1.9 \pm 0.5$ & $2.1 \pm 0.6$ & NS \\
\hline \multirow[t]{2}{*}{$\begin{array}{l}\text { Positive (steroid } \\
\text { maintained) }\end{array}$} & $1.7 \pm 0.6$ & $2.0 \pm 0.6$ & NS \\
\hline & $\begin{array}{l}\text { Blood pressure pre- } \\
\text { transplant (mmHg) }\end{array}$ & $\begin{array}{c}\text { Blood pressure } \\
\text { post-transplant } \\
(\mathrm{mmHg})\end{array}$ & \\
\hline $\begin{array}{l}\text { Negative (steroid } \\
\text { withdrawal) }\end{array}$ & $137 / 77$ & $131 / 72$ & NS \\
\hline $\begin{array}{l}\text { Borderline (steroid } \\
\text { maintained) }\end{array}$ & $128 / 69$ & $130 / 76$ & NS \\
\hline
\end{tabular}

LDL: low- density lipoprotein; NS: non-significant. 